\title{
Directed Evolution of New and Improved Enzyme Functions Using an Evolutionary Intermediate and Multidirectional Search
}

Joanne L. Porter, ${ }^{\dagger}{ }^{\dagger}$ Priscilla L. S. Boon, ${ }^{\dagger}$ Tracy P. Murray, ${ }^{\dagger}$ Thomas Huber, ${ }^{\dagger}$ Charles A. Collyer, ${ }^{\ddagger}$ and David L. Ollis ${ }^{*}$,

\author{
${ }^{\dagger}$ Research School of Chemistry, Australian National University, Canberra, Australian Capital Territory 2601, Australia \\ ${ }^{\ddagger}$ School of Molecular Bioscience, University of Sydney, Sydney, New South Wales 2006, Australia
}

\section{Supporting Information}

ABSTRACT: The ease with which enzymes can be adapted from their native roles and engineered to function specifically for industrial or commercial applications is crucial to enabling enzyme technology to advance beyond its current state. Directed evolution is a powerful tool for engineering enzymes with improved physical and catalytic properties and can be used to evolve enzymes where lack of structural information may thwart the use of rational design. In this study, we take the versatile and diverse $\alpha / \beta$ hydrolase fold framework, in the form of dienelactone hydrolase, and evolve it over three unique sequential evolutions with a total of 14 rounds of screening to generate a series of enzyme variants. The native enzyme has a low level of promiscuous activity toward p-nitrophenyl

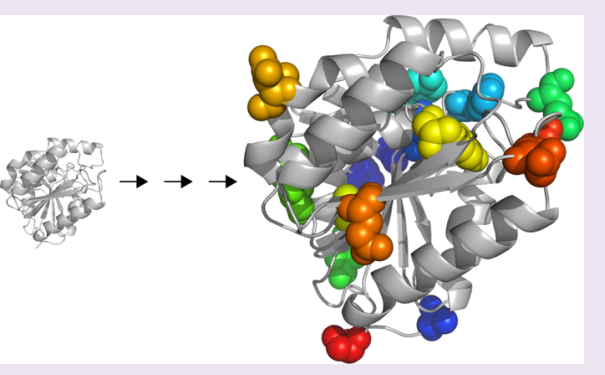
acetate but almost undetectable activity toward larger $p$-nitrophenyl esters. Using $p$-nitrophenyl acetate as an evolutionary intermediate, we have generated variants with altered specificity and catalytic activity up to 3 orders of magnitude higher than the native enzyme toward the larger nonphysiological $p$-nitrophenyl ester substrates. Several variants also possess increased stability resulting from the multidimensional approach to screening. Crystal structure analysis and substrate docking show how the enzyme active site changes over the course of the evolutions as either a direct or an indirect result of mutations.

\begin{abstract}
A lthough enzymes are remarkable catalysts, their use in many practical applications is limited by the availability of large quantities of stable enzyme with appropriate catalytic properties. Directed evolution can be usefully employed to enhance enzyme stability and alter substrate specificity to engineer tailored biocatalysts. ${ }^{1-4}$ Mutations are introduced at the genetic level yielding a library of variant enzymes that are screened for a desired trait. It is extremely difficult to evolve an enzyme to have a completely new catalytic function; rather an existing and weak promiscuous activity can be exploited and greatly enhanced. Here we report the multistep process by which the substrate specificity of the enzyme dienelactone hydrolase (DLH) has been altered so that it can act upon ester and lipase substrates with long alkyl chains (C4-C12), while turnover of these substrates by the native enzyme is virtually undetectable.

$\mathrm{DLH}$ is one of the many hydrolytic enzymes that has an $\alpha / \beta$ hydrolase fold. While these enzymes do not necessarily share any significant sequence similarity, they do share remarkably similar tertiary structures and a preserved arrangement of catalytic residues, suggesting possible evolution from a common ancestor. ${ }^{5,6}$ The catalytic residues include a highly conserved triad, consisting of a nucleophile (serine, cysteine, or aspartic acid), an acid, and a histidine. The most significant variations among these enzymes are in the residues responsible for substrate binding. As well as hydrolases, enzymes exhibiting this characteristic fold can include proteases, esterases, peroxidases, lipases, and dehalogenases. ${ }^{7}$ It appears as if nature has taken an
\end{abstract}

effective catalytic core and altered the substrate binding domain to cater to a variety of substrates. We have set out to do the same with DLH from Pseudomonas knackmussii one of the simplest of known $\alpha / \beta$ hydrolase fold enzymes. ${ }^{8-10}$

$\mathrm{DLH}$ is $25.5 \mathrm{kDa}$ monomeric protein and is the third of four enzymes that constitute the chlorocatechol branch of the $\beta$-ketoadipate pathway. ${ }^{11-14}$ It consists of 236 amino acids that form eight strands of $\beta$-sheet in the core of the enzyme with one buried $\alpha$-helix and six others on the solvent accessible surface. ${ }^{9}$ The catalytic triad consists of a cysteine (C123), an aspartic acid (D171), and a histidine (H202). ${ }^{15}$ Previous studies have shown that mutation of the nucleophile to a serine (C123S) gives an enzyme with altered isomerase activity toward the physiological substrate. ${ }^{16,17}$ The DLH C123S variant was the starting point for the present study, referred to as native $\mathrm{DLH}$ in the remainder of this report, since unlike the native cysteine, serine is not prone to oxidation. It has low but detectable activity toward simple substrates like $p$ nitrophenyl acetate and $\alpha$-naphthyl acetate but almost nonexistent activity toward $p$-nitrophenyl butyrate and longer chain $p$-nitrophenyl esters, too low to detect in crude lysates during library screening.

We set out to generate DLH variants that have good activity toward long chain $p$-nitrophenyl esters and in doing so alter the

\footnotetext{
Received: October 7, 2014

Accepted: November 24, 2014

Published: November 24, 2014
} 
activity of the enzyme from a simple hydrolase to an esterase or lipase. These are the most highly utilized biocatalysts where they are commonly found in cleaning products and have applications in industries such as food processing and pharmaceutics. ${ }^{18,19}$ Our intention was to first increase the activity toward $p$-nitrophenyl acetate with the understanding that this would also give increased activity toward esters with longer chains so that large libraries could then be screened for activity toward $p$-nitrophenyl butyrate. At various stages in this process, we monitored DLH expression and solubility. These latter experiments were done because we were aware that increased activity in crude lysates could be achieved through improvements in expression or solubility. Additionally, we were aware that active site mutations could compromise the overall stability of the protein and thus hinder attempts to evolve for further increases in activity; thus we also monitored changes in stability.

\section{RESULTS AND DISCUSSION}

Overview. Three sequential directed evolution experiments were carried out, each consisting of multiple rounds of mutagenesis and screening (Figure 1). The method of library

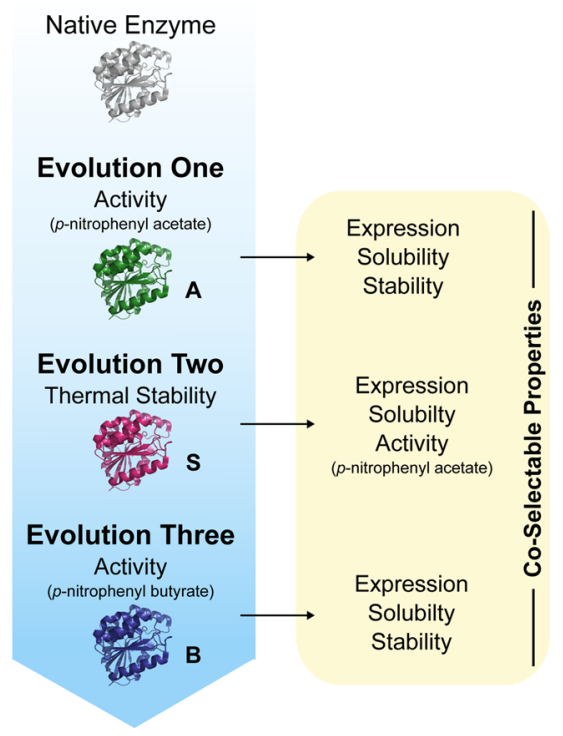

Figure 1. Strategy for the directed evolution of DLH.

screening was different for each evolution, reflecting the aims of the experiment. The first evolution selected for improvements in activity toward $p$-nitrophenyl acetate, a small ester substrate to which the native enzyme possesses a low level of promiscuous activity. Although only one property was targeted, due to the nature of the screens, improvements in other physical properties would also give rise to improvements in the targeted property, termed coselectable properties. For example, when we select for improved activity, a mutation improving protein expression, even a silent mutation, would manifest as a positive variant since the presence of more enzyme would increase substrate turnover rate. Hence, improvements in expression, solubility, or stability would provide positive results under the screening conditions for improved enzymatic activity (see Methods). Over the course of this evolution, especially during the later rounds, several surface mutations were accumulated. These mutations were assumed to be stabilizing since SDSPAGE analysis (Supporting Figure S1, Supporting Information) showed no significant changes in expression or solubility, although SDS-PAGE gives only a gross estimate of these properties so slight changes may have occurred.

It is widely acknowledged that a more stable protein has a greater capacity to evolve. ${ }^{20-22}$ The additional stability confers the ability to accommodate the often destabilizing mutations that are required for the acquisition of new and improved enzyme functions. Given that stabilizing mutations were acquired during the first set of evolution experiments and the proven importance of protein stability on evolvability, a second set of evolution experiments were conducted to produce a variant with increased thermal stability. This variant was then used as the starting point for the third and final set of evolution experiments to increase the activity and specificity of DLH toward longer chain $p$-nitrophenyl esters. At this point, the level of activity toward the longer chain esters was adequate for highthroughput library screening.

Like the first evolution, the second and third also had coselectable properties that gave rise to improved variants under the primary selection pressure. The stability of mutants during the second evolution was determined based on residual activity after incubation at an elevated temperature; therefore more active variants could also be identified under this screen. The coselectable traits for the third evolution toward $p$ nitrophenyl butyrate were the same as the $p$-nitrophenyl acetate evolution. As with the first evolution, there appeared to be minimal change to expression or solubility based on SDSPAGE analysis (Supporting Figures S2 and S3, Supporting Information). To summarize, the following study presents a three-dimensional search along three consecutive evolutionary trajectories to evolve DLH for improved activity, substrate specificity, and stability.

Directed Evolution toward an Evolutionary Intermediate. Six rounds of directed evolution were carried out to improve the activity of DLH toward $p$-nitrophenyl acetate (Supporting Table S1, Supporting Information). Random mutagenesis in the form of error-prone PCR was used to generate genetic diversity, and libraries of 5000 variants were screened. Library and screening details for all three evolutions are given in Table 1. Three important active site mutations, $\mathrm{Q} 35 \mathrm{H}, \mathrm{F} 38 \mathrm{~L}$, and Y145C, were found individually in generation one and combined through shuffling in generation two (Figure 2). The combined mutations in the generation two variant (A-2) gave a 16-fold improvement in catalytic activity compared with the native enzyme but reduced the stability of the enzyme as shown by the $5.0 \pm 0.4{ }^{\circ} \mathrm{C}$ decrease in melting temperature. Melting temperature was determined from the residual activity of the enzymes following heat treatment; Supporting Figure S4, Supporting Information, shows the residual activity plots, and Figure 3 shows the melting temperatures determined by analysis based upon a Hill type equation (see Methods).

Mutations and kinetic constants for the characterized variants from this and following evolutions are shown in Tables 2 and 3. The $k_{\text {cat }}$ of the best variants from generations 2,4 , and 6 (A-2, A-4, and A-6) increased steadily, but absence of the F38L mutation in the A-4 variant caused an increase in $K_{\mathrm{m}}$ and consequential drop in $k_{\text {cat }} / K_{\mathrm{m}}$. The F38L mutation returned in the A-6 variant, alongside the E199G and S208G mutations, all of which were fully conserved in the final generation. Residues 199 and 208 are on the loop containing H202 of the catalytic triad, and mutation of these residues to glycine gives improved activity. Over the subsequent generations, stabilizing surface 
Table 1. Experimental Details for Library Generation and Screening

\begin{tabular}{|c|c|c|c|c|c|c|c|c|c|}
\hline & \multirow[b]{2}{*}{$\begin{array}{l}\text { number } \\
\text { of rounds }\end{array}$} & & \multicolumn{2}{|c|}{ mutation rate } & \multirow[b]{2}{*}{$\begin{array}{l}\text { theoretical } \\
\text { library size }\end{array}$} & \multirow[b]{2}{*}{ primary screen method } & \multirow{2}{*}{$\begin{array}{c}\text { primary } \\
\text { screen } \\
\text { size }^{a}\end{array}$} & \multirow[b]{2}{*}{ secondary screen method } & \multirow[b]{2}{*}{$\begin{array}{l}\text { secondary } \\
\text { screen size }\end{array}$} \\
\hline & & & $\begin{array}{l}\text { base } \\
\text { pairs }\end{array}$ & $\begin{array}{l}\text { amino } \\
\text { acids }\end{array}$ & & & & & \\
\hline evolution one & 6 & & 2 & 1 & 1400 & activity assay (96-well plates) & 5000 & activity assay (96-well plates) & 250 \\
\hline evolution two & 3 & & 3 & $1-2$ & 3500 & $\begin{array}{l}\text { activity after heat treatment } \\
\text { (filter paper) }\end{array}$ & 10000 & $\begin{array}{l}\text { residual activity after heat } \\
\text { treatment (96-well plates) }\end{array}$ & 350 \\
\hline \multirow[t]{2}{*}{ evolution three } & 5 & stage 1 : rounds $1-3$ & 4 & 2 & 8500 & activity assay (filter paper) & 24000 & activity assay (96-well plates) & 350 \\
\hline & & stage 2 : rounds $4-5$ & 2 & 1 & 1400 & activity assay (96-well plates) & 5000 & activity assay (96-well plates) & 250 \\
\hline
\end{tabular}

${ }^{a}$ Triple the theoretical library size should be screened in order the achieve $95 \%$ library coverage.

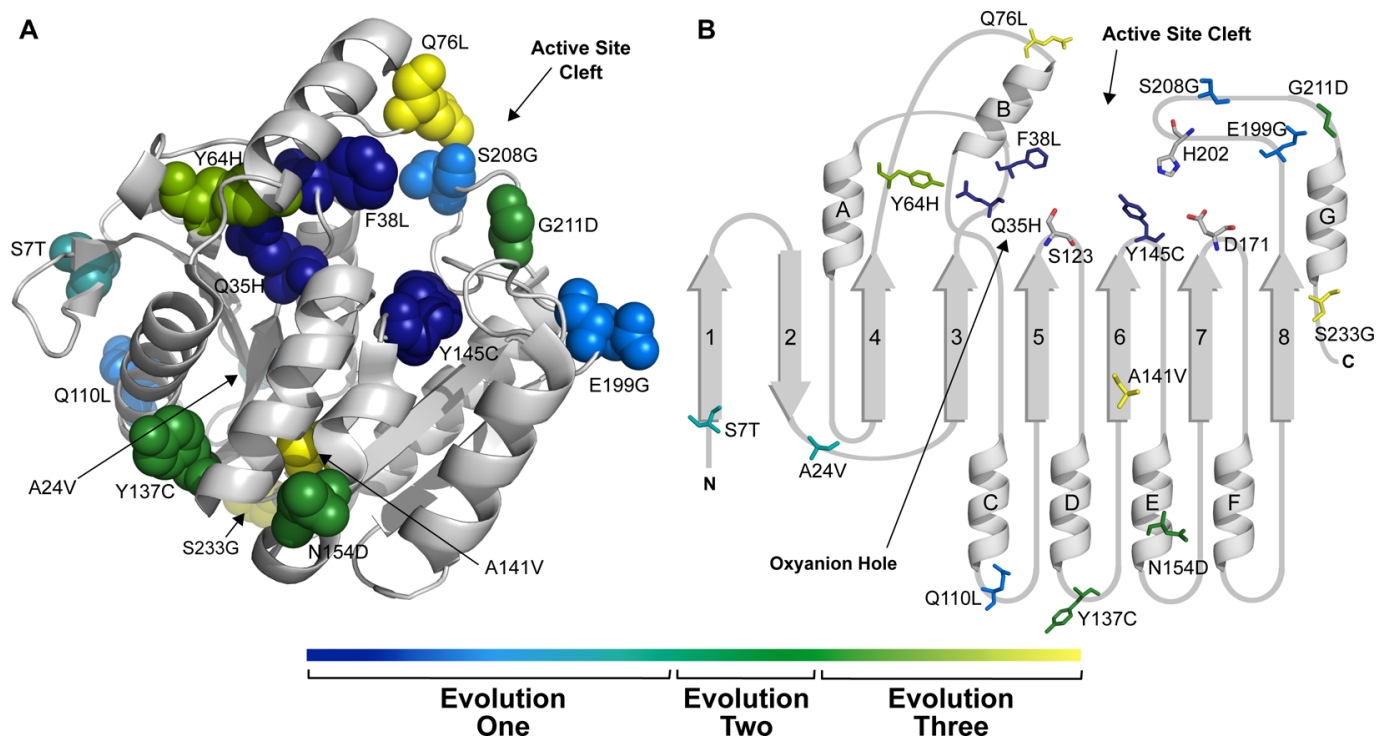

Figure 2. Location of mutations accumulated over three sequential evolutions shown (A) in 3-D and (B) as a 2-D schematic.

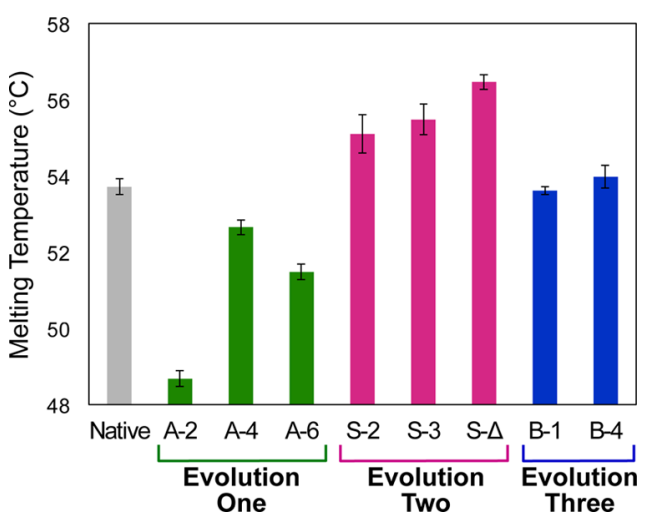

Figure 3. Melting temperature of native and evolved DLH variants calculated from residual activity.

mutations compensated for the loss of stability introduced by the active site changes enabling the enzyme to accommodate and combine the activity enhancing mutations, which were fully conserved in the final round of evolution. Several mutations (S7T, A24V, G17D, D53N, A65G, and Q110L) thought to provide neutral or stabilizing effects appeared multiple times over the generations, of these the only one to be fully conserved was Q110L. The best variant after six generations (A-6) of evolution had a 45 -fold increase in $k_{\text {cat }} / K_{\mathrm{m}}$ for the hydrolysis of $p$-nitrophenyl acetate. It also showed improved activity toward the larger $p$-nitrophenyl butyrate substrate with an 85-fold improvement in $k_{\mathrm{cat}} / K_{\mathrm{m}}$. Modest increases in substrate specificity were indicated for both substrates with $K_{\mathrm{m}}$ values of $34 \pm 2$ and $146 \pm 16 \mu \mathrm{M}$ for $p$-nitrophenyl acetate and butyrate compared with $40 \pm 2$ and $175 \pm 4 \mu \mathrm{M}$ for the native enzyme.

Site saturation mutagenesis (SSM) was conducted on the A-6 variant at sites 38 and 145 individually and at sites 85 and 88 jointly. It was suspected that alternative residues could better fill the role of the F38L and Y145C mutations obtained during evolution one. Residues Y85 and W88 were chosen because they are involved in substrate binding. None of these sites yielded beneficial mutations; a scarce number of neutral mutations were found, but most were detrimental (data not shown).

Improving the Thermostability and Evolvability of DLH. Three rounds of evolution were carried out to improve the stability of DLH before we attempted to evolve activity toward larger substrates (Supporting Table S2, Supporting Information). The A-6 variant from the $p$-nitrophenyl acetate evolution $\left(T_{\mathrm{m}}=51.5 \pm 0.2{ }^{\circ} \mathrm{C}\right)$ was used as a template for library generation. Libraries with sizes in excess of 10000 variants were screened for each round with an average of 1-2 amino acid mutations per variant. The best variants from the second and third generations (S-2 and S-3) had improved thermal stability and improved activity toward both substrates. These variants both contained the mutations Y137C, N154D, and G211D. The latter mutation is to the loop containing the histidine of the catalytic triad and contributed to the increased 
ㅎํㅇ $+n a=\exists n$<smiles>[Li][Te]</smiles><smiles>[Li]</smiles>

్ֻలై

光光光录

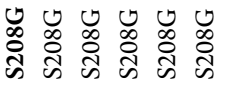

๖ু

वे

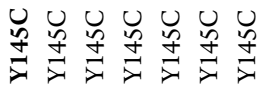

$$
\begin{aligned}
& \frac{2}{4}
\end{aligned}
$$

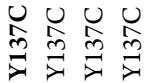

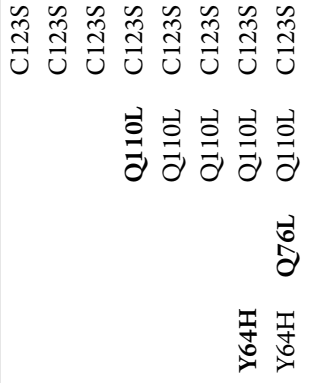

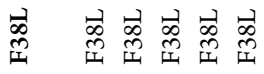

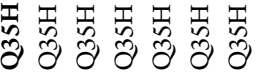$$
\text { 安 }
$$$$
\text { 온 }
$$

药

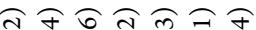

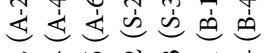

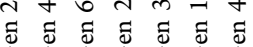
पु

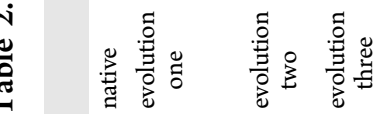

activity. The only difference between the S-2 and S- 3 variants was the addition of a single mutation $\mathrm{K} 234 \mathrm{~N}$ at the $\mathrm{C}$-terminus of the S-3 variant. From crude lysate assays during screening, it appeared the S-3 variant had approximately $2{ }^{\circ} \mathrm{C}$ higher melting temperature than the S-2 variant (data not shown). This turned out not to be the case when melting temperature was measured more accurately using purified protein; the melting temperatures were in fact very similar and within error. The melting temperatures determined from residual activity after $15 \mathrm{~min}$ heat exposure using purified protein were $55.1 \pm 0.5$ and $55.5 \pm 0.4{ }^{\circ} \mathrm{C}$ giving improvements of $3.6 \pm 0.7$ and $4.0 \pm$ $0.6^{\circ} \mathrm{C}$, respectively (Figure 3 ).

Residues K234, K235, and P236 are highly flexible and as a result have always been disordered in crystal structures; therefore determination of their influence on the properties of the enzyme is difficult. ${ }^{9,23,24}$ In response to the K234N mutation that initially appeared to give increased activity, a variant was engineered with these terminal residues deleted (S- $\Delta$ ). Characterization of this enzyme showed it had greater stability $\left(T_{\mathrm{m}} 56.5 \pm 0.2{ }^{\circ} \mathrm{C}\right)$ and solubility (Supporting Figure S2, Supporting Information) than the full-length enzyme while retaining similar kinetic properties.

Altering Substrate Specificity. Screening for improved activity toward $p$-nitrophenyl butyrate was done in two stages (Supporting Table S3, Supporting Information). Variants from generations one and four were characterized. The generation one variant (B-1) contained a single mutation additional to the mutations conserved from the S-2 variant that was used as a template for this evolution. The Y64H mutation was fully conserved throughout five rounds of directed evolution; it is located in the core of the enzyme separated from the active site by residues of the oxyanion hole (A34, Q35(H), E36, and I37). The B-1 variant had improved substrate specificity toward $p$-nitrophenyl butyrate with a $K_{\mathrm{m}}$ of $59 \pm 2 \mu \mathrm{M}$ compared with $175 \pm 4 \mu \mathrm{M}$ for the native enzyme. The Y64H mutation gave an increase in $k_{\text {cat }}$ for both substrates, but the increase was more significant for the hydrolysis of $p$-nitrophenyl butyrate with a $k_{\text {cat }} / K_{\mathrm{m}}$ value of $(2.1 \pm 0.1) \times 10^{4} \mathrm{M}^{-1} \mathrm{~s}^{-1}$, which is 3 orders of magnitude greater than the native rate of $21 \pm 1 \mathrm{M}^{-1} \mathrm{~s}^{-1}$.

The best variant from the fourth generation (B-4) contained the additional mutations Q76L, A141V, and S233G and incorporation of glutamine $(237 \mathrm{Q})$ at the C-terminus replacing the first of two stop codons in the DNA sequence. These mutations were thought to be primarily stabilizing; however they also caused a change in the kinetic profile of the enzyme. The Q76L mutation was thought to be responsible for this since it is solvent exposed at the opening of the active sight. It caused a slight decrease in substrate specificity but a $20 \%$ increase in $k_{\text {cat }}$ for the hydrolysis of $p$-nitrophenyl butyrate compared with the B-1 variant and an overall 400-fold increase compared with the native enzyme. These mutations were conserved in the fifth generation where the evolution appeared to converge.

Addition of $237 \mathrm{Q}$ to the $\mathrm{C}$-terminus of the enzyme was unexpected since the terminal three residues are highly flexible and do not form part of any secondary or tertiary structural elements. It is likely that these residues are the consequence of a nucleotide sequence necessary for the translation of the following enzyme in the operon. The genes $(c l c A, c l c B$, $c l c D$, and $c l c E$ ) encoding the four enzymes of the modified ortho-cleavage branch of the $\beta$-ketoadipate pathway are located together in an operon (Supporting Figure S5, Supporting Information). The $c l c E$ gene encoding maleylacetate reductase is immediately after the $c l c D$ gene encoding $\mathrm{DLH}$; consequently 


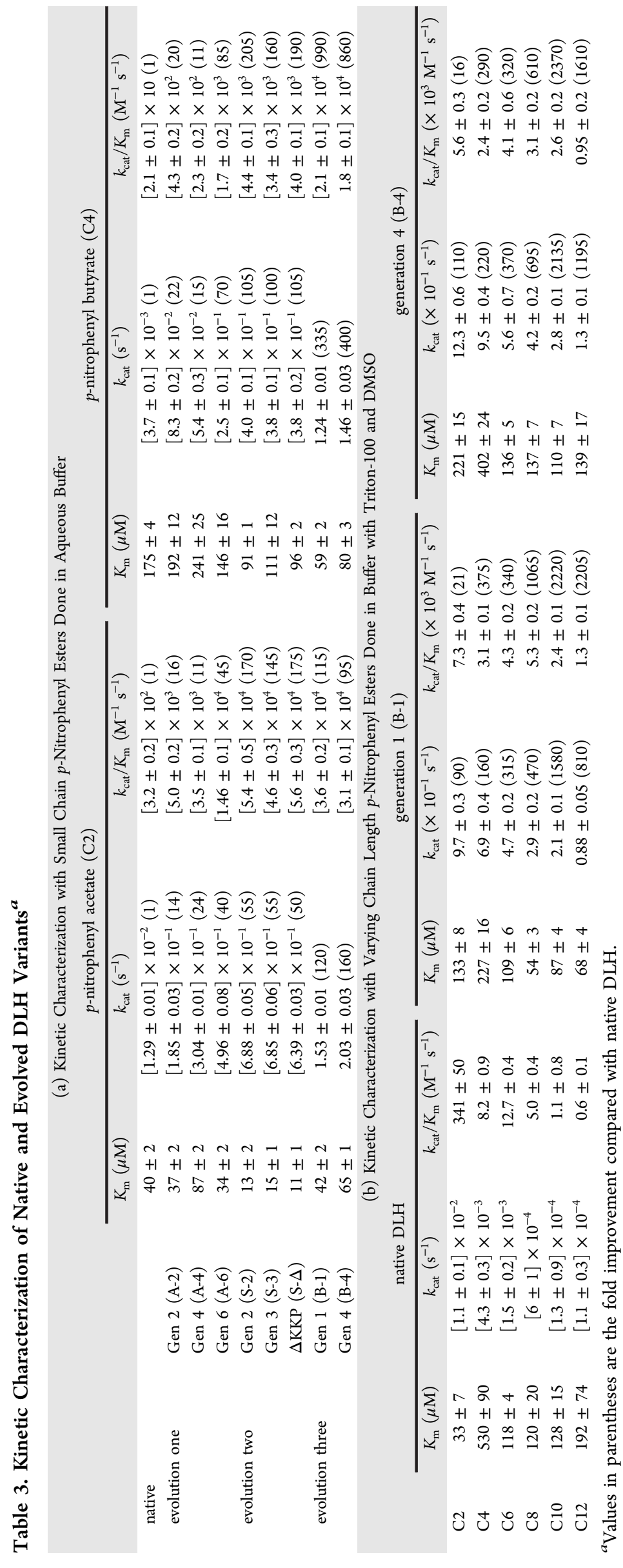




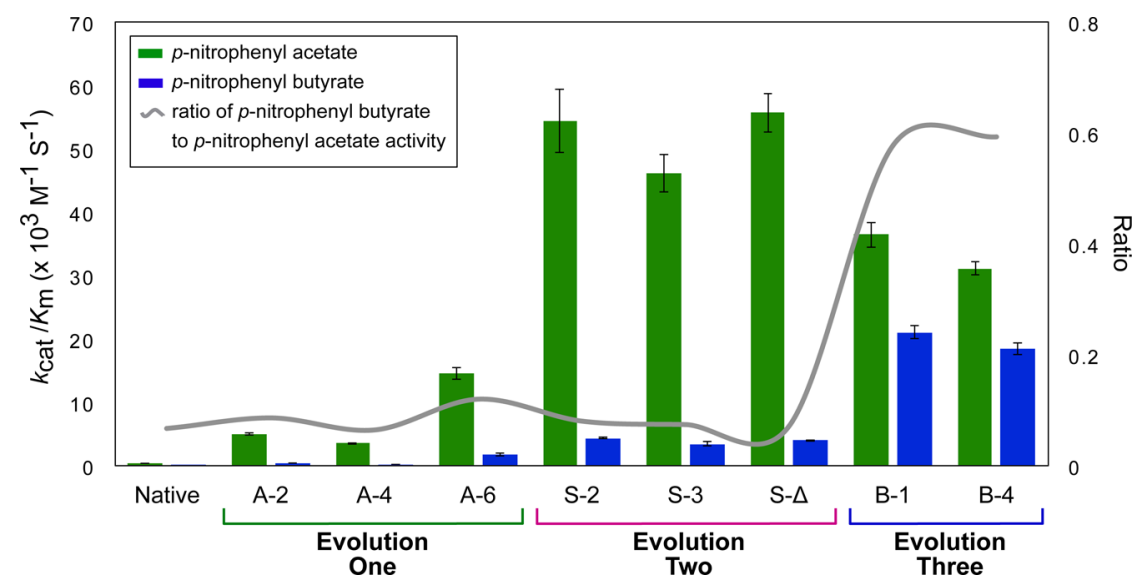

Figure 4. Specific activity toward two substrates over three evolutions and the ratio of $k_{\mathrm{cat}} / K_{\mathrm{m}}$ for $p$-nitrophenyl butyrate to $p$-nitrophenyl acetate.

there is a ribosome binding site and suspected proteolytic cleavage site at the end of the DLH gene. ${ }^{25}$ We suspected that the nucleotides encoding the three C-terminal DLH residues are inconsequential to the function of DLH but are necessary for translation of maleylacetate reductase. Deletion of the three C-terminal residues, as in the S- $\Delta$ variant, did in fact improve the stability and solubility (Supporting Figure S2, Supporting Information) of the enzyme lending credence to this hypothesis.

Enzymatic Activity toward Longer Acyl Chain pNitrophenyl Esters. The native enzyme has low activity toward both $p$-nitrophenyl acetate and butyrate with $k_{\text {cat }} / K_{\mathrm{m}}$ values of $320 \pm 10$ and $21 \pm 1 \mathrm{M}^{-1} \mathrm{~s}^{-1}$, respectively (Figure 4). All characterized variants have improved activity compared with the native enzyme for these two substrates. The activity of the native enzyme along with B-1 and B-4 variants from the third evolution were measured toward the larger p-nitrophenyl hexanoate (C6), octanoate (C8), decanoate (C10), and dodecanoate (C12) substrates (Table $3 b$ ). This was done in the presence of $0.2 \%$ Triton X-100 and $4 \%$ dimethyl sulfoxide (DMSO) to bolster substrate solubility. Kinetic parameters of the enzymes with the smaller C2 and $\mathrm{C} 4$ substrates were measured in both buffer compositions for consistency.

The addition of Triton X-100 and DMSO to the assay buffer changed the $K_{\mathrm{m}}$ values for the smaller substrates quite drastically, especially in the case of $p$-nitrophenyl butyrate. In aqueous buffer, the $K_{\mathrm{m}}$ values for the native, B-1, and B-4 variants were $175 \pm 4,59 \pm 2$, and $80 \pm 3 \mu \mathrm{M}$, while in the presence of the detergent and organic solvent, the $K_{\mathrm{m}}$ values increased to $530 \pm 90,227 \pm 16$, and $402 \pm 24 \mu \mathrm{M}$, respectively. The native enzyme had the best activity toward $p$-nitrophenyl acetate and almost negligible activity toward the larger $p$-nitrophenyl ester substrates (C6-C12). The B-1 and B-4 variants had good enzymatic activity toward all substrates tested. The B-1 variant had $k_{\mathrm{cat}} / K_{\mathrm{m}}$ of $(1.3 \pm 0.1) \times 10^{3} \mathrm{M}^{-1} \mathrm{~s}^{-1}$ for the hydrolysis of $p$-nitrophenyl dodecanoate, a 2205-fold improvement for this substrate and a 3.8 -fold improvement compared with the activity of the native enzyme toward its favored $p$-nitrophenyl acetate substrate.

Structural Changes to the DLH Active Site over Multiple Evolutions. The mutations Q35H, F38L, Y64H, E199G, S208G, and G211D are either in or immediately surrounding the enzyme active site. Singularly the Q35H mutation gave the largest improvement in activity. In the native enzyme Q35 hydrogen bonds with the backbone of E36 and L63, these hydrogen bonds make the local structure more rigid and stabilize the active site. The mutation of glutamine to histidine is subtle in relation to size so the packing and hydrophobic interactions of the surrounding residues remain undisturbed. However, even with the crystal structures and substrate docking simulations (see Methods), it was hard to decipher the exact role played by the $\mathrm{Q} 35 \mathrm{H}$ mutation in facilitating the observed increased activity.

Mutations F38L and Y145C enlarge the enzyme active site in favor of the larger nonphysiological substrates rather than the smaller and more polar dienelactones. The substitution of phenylalanine to leucine at position 38 is not drastic because both residues are hydrophobic, but leucine is smaller so the change increased the size of the active site. The substitution of tyrosine to cysteine at position 145 was also exchanging a large residue for a smaller one, thereby increasing the size of the active site. Site-saturation mutagenesis (SSM) was performed at this position with the belief that other residues such as serine or alanine could fill this role better than cysteine, especially since in the A-6 variant it was oxidized. However, SSM yielded no variants with activity equal to or better than the cysteine found in the first evolution (data not shown). Interestingly during evolution two, the G211D mutation appeared, and in the crystal structure of this variant and all subsequent variants, the cysteine was not oxidized (Figure 5a). Mutation of glycine to aspartic acid at position 211 caused the amide nitrogen of S203 to flip in order to avoid a clash between the carboxylic acid of G211D and the backbone carbonyl oxygen of H202. The new position of the $\mathrm{H} 202$ carbonyl oxygen would cause a clash with the oxidized cysteine at position 145 and therefore prevents oxidation.

Other mutations important in enhancing the activity of DLH are the mutations to loop 14 (residues 198-212) containing $\mathrm{H} 202$ of the catalytic triad (Figure 5a). Loop 14 functions as a cover for the active site, and mobility of this loop is important to facilitate substrate binding and subsequent release of product but more importantly to allow the crucial accessibility of $\mathrm{H} 202$ during catalysis. The proposed mechanism for the DLH catalyzed hydrolysis of the native dienelactone substrates sees the enolate-enzyme intermediate deprotonate the water molecule itself to generate the nucleophile needed for release of the product and regeneration of free enzyme. ${ }^{26}$ The mechanism for the hydrolysis of $p$-nitrophenyl esters is thought to follow that of the serine proteases whereby the catalytic 

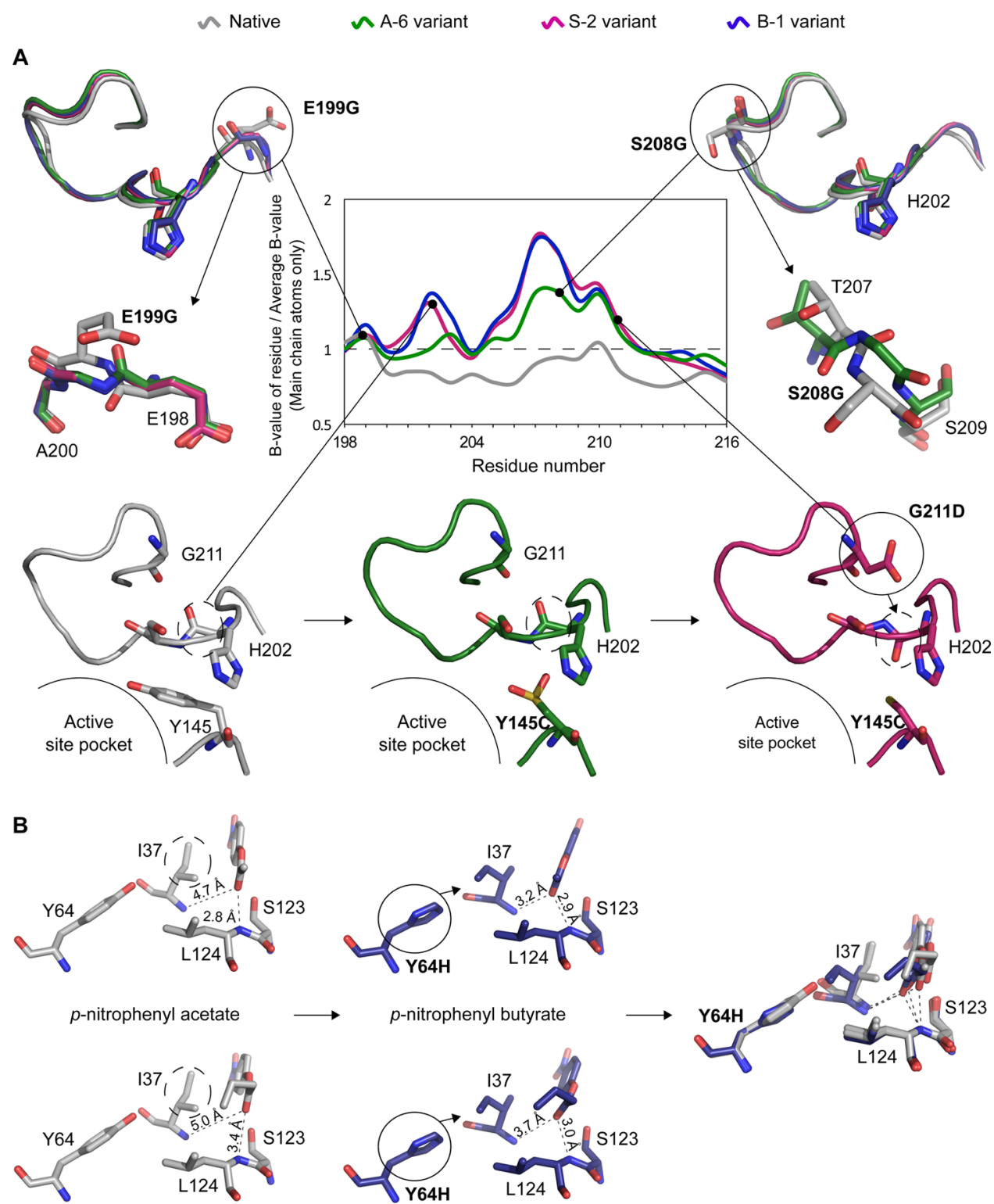

Figure 5. Evolution of the DLH active site showing (A) changes in the flexibility of the active site loop and (B) change in substrate positioning caused by the $\mathrm{Y} 64 \mathrm{H}$ mutation.

histidine is responsible for generation of the hydroxide nucleophile during the catalysis and not the substrate itself. ${ }^{27}$ This difference in mechanism explains the accumulation of mutations on loop 14 and the evolution of increased flexibility allowing H202 more freedom where it was not required before.

The mutations E199G and S208G see the replacement of larger residues for smaller and more flexible glycine residues, with one either side of $\mathrm{H} 202$. The substitution of two glycine residues into the loop increased its flexibility and subsequently made the histidine more accessible during catalysis. The normalized B-values for the $\mathrm{H} 202$ side chain of 0.77 and 1.01 for the native and generation A- 6 variant represent a $30 \%$ increase in relative thermal motion in the crystallographic environment, which is consistent with increased mobility.

The mutation of tyrosine to histidine at position 64 gave improved activity toward both substrates but an overall loss in specific activity toward $p$-nitrophenyl acetate due to an increase in $K_{\mathrm{m}}$. However, for the larger substrate, the $K_{\mathrm{m}}$ decreased with an improved $k_{\text {cat }}$ giving 3 orders of magnitude improvement in $k_{\text {cat }} / K_{\mathrm{m}}$ for the hydrolysis of $p$-nitrophenyl butyrate. Residue 64 is separated from the active site by the residues of the oxyanion hole. Both the original tyrosine and substituted histidine form hydrogen bonds with the carbonyl oxygen of residue I37. During catalysis, the enzyme-acyl tetrahedral intermediate is stabilized by the backbone amide of I37 and L124 in the oxyanion hole. ${ }^{26}$ In the crystal structures, the I37 side chain protrudes into the active side in a decidedly similar position for the native and evolved enzymes. Substrate docking experiments showed that the substrate is prevented from binding tightly in this pocket due to the position of this side chain. Mutation of tyrosine to histidine at position 64 in the B-1 variant creates space behind residue 37 enabling the side chain to rotate upon substrate binding (Figure $5 b$ ). This rotation opens the active site creating more space for the bulkier nonphysiological $p$-nitrophenyl ester substrates allowing the carbonyl oxygen of the substrate to hydrogen bond with residues I37 and L124 in the oxyanion hole. The proximity of the polar part of the substrate to the oxyanion hole will aid in stabilization of the 


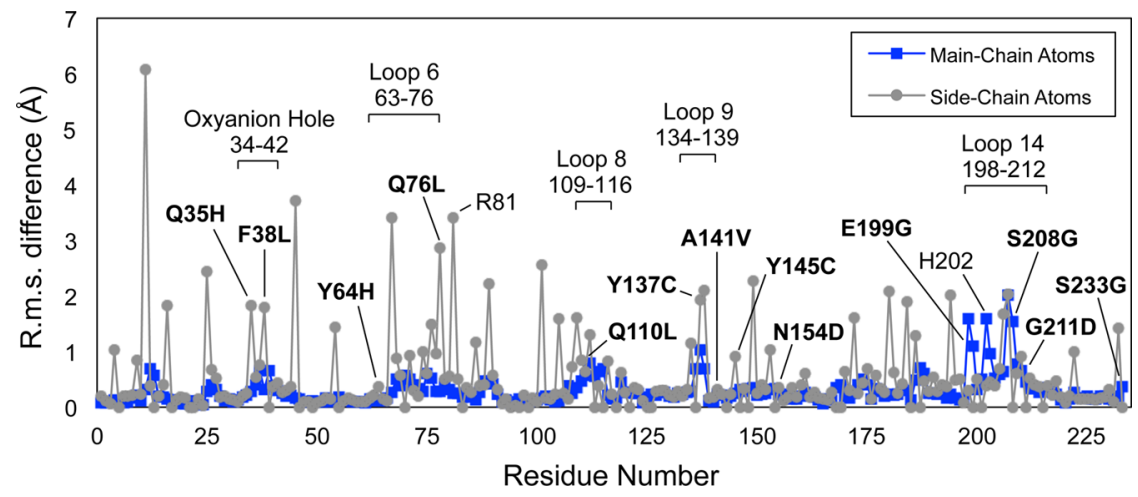

Figure 6. RMS displacement plot for the superimposition of the native and B-4 DLH variant for both main-chain and side-chain atoms.

enzyme-substrate intermediate during catalysis and facilitate release of products and regeneration of free enzyme thereby increasing the catalytic rate. Although this mutation gave the B-1 variant an increased $k_{\text {cat }}$ for all tested substrates, the specificity is lowered for the smaller $p$-nitrophenyl acetate substrate but significantly increased for the larger $p$-nitrophenyl butyrate substrate.

Overall Structural Changes. The overall structure of native DLH and the evolved variants for which crystal structures were obtained are decidedly similar. The RMS displacements for the superposition of variant structures with the native are $0.84,0.86,0.81,0.81$, and $0.84 \AA$ for the A-6, S-2, S-3, B-1, and B-4 variants. When the superposition was limited to main-chain atoms, the RMS displacements were $0.40,0.41$, $0.40,0.41$, and $0.39 \AA$ A respectively. The RMS displacement plot for residues of the native enzyme superimposed with the B-4 variant highlights structural regions with the most variation (Figure 6). Unsurprisingly the most significant change to the protein backbone occurs at loop 14 where the previously discussed mutations (E199G, S208G, and G211D) around the catalytic $\mathrm{H} 202$ alter the local structure and increase the flexibility. Other regions showing slight change in structure include the oxyanion hole where mutations $\mathrm{Q} 35 \mathrm{H}$ and $\mathrm{F} 38 \mathrm{~L}$ occur and loop 8 and 9 featuring mutations Q110L and Y137C.

\section{CONCLUSIONS}

Three consecutive sets of directed evolution experiments have been used to successfully improve and alter the specificity of DLH. The low level of promiscuous activity exhibited toward $p$-nitrophenyl acetate has been increased such that the $k_{\text {cat }} / K_{\mathrm{m}}$ of evolved variants approaches values typically observed in enzymes, with the best variant showing a 175 -fold improvement over native DLH. The target substrate of the third evolution can be hydrolyzed by variants at a rate 3 orders of magnitude greater than the native enzyme. Additionally the evolved variants have significant activity toward a range of larger $p$-nitrophenyl esters, while the native enzyme has virtually undetectable levels of activity toward substrates with acyl chains longer than $\mathrm{C} 4$. The $k_{\mathrm{cat}}$ and $k_{\mathrm{cat}} / K_{\mathrm{m}}$ of the best variants from evolution three (B-1 and B-4) toward the $\mathrm{C} 10$ and $\mathrm{C} 12$ substrates were in excess of 2000 -fold greater than the native enzyme, with the best being a 2370-fold improvement in $k_{\mathrm{cat}} / K_{\mathrm{m}}$ for the hydrolysis of $p$-nitrophenyl decanoate (C10) by the B-4 DLH variant.

The combination of seven active site mutations gave rise to the changes in kinetic parameters. The mutations Q35H, F38L, and Y145C gave improved activity toward all $p$-nitrophenyl ester substrates, while the $\mathrm{Y} 64 \mathrm{H}$ mutation was specific for larger $p$-nitrophenyl esters. These mutations are in or around the oxyanion hole, which is responsible for stabilization of the enzyme-substrate intermediate complex. It is clear from the magnitude of the observed increase in catalytic rate and the position of these mutations that the oxyanion hole is better able to serve its purpose in the evolved enzymes. Three important mutations were accumulated in the loop containing the histidine of the catalytic triad, E199G, S208G, and G211D. These mutations increase the flexibility of the loop resulting in increased movability of $\mathrm{H} 202$ and therefore improved accessibility during catalysis for its role in generation of the nucleophile leading to release of the second product and regeneration of free enzyme. Although most of the esterases and lipases of the $\alpha / \beta$ hydrolase fold family have rather elaborate substrate binding domains, ${ }^{6}$ DLH has a minimal stretch of peptide responsible for substrate binding. Stretches of helix B (Figure 2) and sections of loop 14 are involved in substrate binding in $\mathrm{DLH}$ while in acetylcholinesterase a large domain serves the same purpose. ${ }^{28}$ We have evolved DLH to be capable of hydrolyzing esterase and lipase substrates even though the residues responsible for substrate binding are minimal. This may account for the evolved broad substrate specificity.

Additional to the significant activity improvements and altered specificity of the evolved variants, the stability was maintained throughout the three evolutions allowing the rapid accumulation of active site mutations. Furthermore, the three C-terminal residues have been shown to be superfluous to the structure and function of DLH and are actually a source of instability and most likely contribute to the initiation of protein unfolding. Deletion of these residues increased not only the stability but also the solubility of the enzyme.

The ease with which enzymes can be modified is crucial to see the continued use of enzyme technology in roles outside of those nature intended. This study confirms the position of directed evolution as a premier approach to engineering enzymes tailored for specific industrial or commercial applications. The use of DLH, a simple and well-characterized enzyme, has enabled a thorough structural and mechanistic investigation of the mutations accumulated over three sequential evolutions. This multidirectional approach has displayed the rapid evolution of improved and altered catalytic properties while maintaining a stable and evolvable protein. This work presents the evolutionary pathway of an enzyme under multiple selection pressures, activity, stability, and specificity, and is of value to our global understanding of evolutionary biology as well as setting a benchmark for the 
future evolution of more complex and less well characterized enzymes.

\section{METHODS}

Library Generation. Error-prone PCR with the addition of manganese $(0.1-0.2 \mathrm{mM})$ was used to introduce mutations into the DLH gene (Supporting Table S5, Supporting Information). The PCR product was digested at $\mathrm{NdeI}$ and EcoRI restriction sites and cloned into the constitutive expression vector $p \mathrm{CY}^{29} 6^{29}$ and then transformed into electrocompetent DH5a Escherichia coli cells. DNA sequencing was performed to test the mutation rate during library construction and following screening for selected individual variants. This was done on an AB 3730xI DNA analyzer (Applied Biosystems) at the ACRF Biomolecular Resource Facility, JCSMR, at the Australian National University.

Screening for Improved $p$-Nitrophenyl Acetate Activity. The transformed cells carrying the library of DLH variants were diluted with ampicillin supplemented LB media and distributed into 96-well plates with 2-3 colony forming units (CFU) per well and grown for $24 \mathrm{~h}$. The mutation rate of this library was one to two base changes per gene leading to an average of one amino acid change per enzyme. For the primary screen, cells were lysed using BugBuster (Novagen) and then assayed for $p$-nitrophenyl acetate activity with monitoring at $405 \mathrm{~nm}$. The top $3-4 \%$ of wells from the primary screen were streaked onto ampicillin supplemented LB agar plates and grown overnight to isolate the individual variants comprising the wells. Six single colonies from each streaked well were inoculated into 96-well plates and subjected to a similar secondary screen. The variants with the highest activity from the secondary screen were isolated, and the DNA was sequenced and used as template for the subsequent round.

Screening for Improved Thermal Stability. The transformed cells were plated on ampicillin supplemented agar plates with approximately 200 colonies per plate and grown overnight at $37^{\circ} \mathrm{C}$. Colonies were blotted onto filter paper (Whatman) and incubated at elevated temperature for $30 \mathrm{~min}$, while the original agar plates were returned to $37{ }^{\circ} \mathrm{C}$ incubation. Following heat treatment, the filter paper with blotted cells was sprayed with a solution containing $0.25 \%(\mathrm{w} / \mathrm{v}$ ) Fast Blue BB, 2\% acetonitrile, $2 \mathrm{mM} \alpha$-naphthyl acetate, $1 \mathrm{mM}$ ethylenediaminetetraacetic acid (EDTA), and $20 \mathrm{mM} \mathrm{4-(2-}$ hydroxyethyl)-1-piperazineethanesulfonic acid (HEPES), $\mathrm{pH}$ 7.0. Variants with greater activity stained the fasted, and the corresponding colonies were selected from the original plates, inoculated into 96-well plates, and grown overnight before being subjected to a secondary screen. The overnight cultures were assayed twice for $p$-nitrophenyl acetate activity, once with prior heat treatment and once with no heat treatment as described above. Heat treatment consisted of $15 \mathrm{~min}$ incubation at elevated temperature followed by $15 \mathrm{~min}$ on ice allowing time for the enzyme to refold before measurement of residual activity. Two aspects were considered when selecting the best variants, those with the greatest residual activity after the heat treatment, providing they also had equal or greater activity at ambient temperature compared with the parent enzyme. DNA from these selected variants was sequenced then used as template for the following round. Three rounds were carried out in this manner with the temperature of heat treatment increased for each round $\left(52,53\right.$, and $\left.55^{\circ} \mathrm{C}\right)$.

Screening for Improved $p$-Nitrophenyl Butyrate Activity. Screening of variant libraries for improved activity toward $p$ nitrophenyl butyrate was carried out in two stages (rounds 1-3 and rounds $4-5$ ). Rounds $1-3$ were done in a similar manner to the thermal stability screening. The amino acid mutation rate of the library was increased from an average of one mutation per enzyme used in the previous evolution to an average of two amino acid changes. Consequently, to achieve $95 \%$ library coverage when screening, the target number of colonies per agar plate was increased to 400 with a total library size of approximately 24000. Blotted colonies were sprayed with the same solution as before only with $\alpha$-naphthyl butyrate as substrate and the addition of $2 \%$ methanol. The best variants were assayed for $p$-nitrophenyl butyrate activity in a secondary screen, the best 10-20 variants were isolated, and the DNA was sequenced and used as template for subsequent rounds. The libraries for rounds 4 and 5 were screened using the same method as the $p$-nitrophenyl acetate screening only with the larger substrate.

Enzyme Expression and Purification. An identical method of expression and purification was used for the native and variant enzymes closely resembling a method previously described. ${ }^{23}$ Consult the Supporting Information for further detail.

Stability Assays. Enzyme melting temperature was determined by measuring the residual activity of purified protein after $15 \mathrm{~min}$ incubation at elevated temperature followed by $15 \mathrm{~min}$ at $4{ }^{\circ} \mathrm{C}$. Enzyme samples were heated using the gradient function on a C1000 thermal cycler (Bio-Rad); 18 data points were collected in triplicate between 30 and $70{ }^{\circ} \mathrm{C}$ for each sample. The melting temperature was determined by fitting the following equation, ${ }^{30}$ derived from the Hill equation, to the data:

$$
a=a_{0}-\left(a_{0} T^{\mathrm{h}}\right) /\left(T_{1 / 2}^{\mathrm{h}}+T^{\mathrm{h}}\right)
$$

Kinetic Characterization. Enzyme kinetics was done using two sets of conditions due to differing solubility of $p$-nitrophenyl ester substrates. The DLH catalyzed hydrolysis of the substrates in both cases was measured by monitoring the release of $p$-nitrophenolate at $405 \mathrm{~nm}$. The substrate concentration was varied, while the enzyme concentration remained constant throughout. Activity toward the more water-soluble $p$-nitrophenyl acetate (C2) and butyrate (C4) substrates was measured in cuvettes $(1 \mathrm{~mL})$ using a CarylE spectrophotometer (Varian) equipped with thermal control at $25{ }^{\circ} \mathrm{C}$. Substrates were dissolved in acetonitrile and used at $1 \%(\mathrm{v} / \mathrm{v})$ in the final assay buffer containing $20 \mathrm{mM}$ HEPES and $1 \mathrm{mM}$ EDTA at $\mathrm{pH}$ 7.5. Activity toward the larger more hydrophobic $p$-nitrophenyl hexanoate (C6), octanoate (C8), decanoate (C10), and dodecanoate (C12) substrates was measured in a 96-well microtiter plate using a SpectraMax M2e plate reader (Molecular Devices) at $25{ }^{\circ} \mathrm{C}$. Substrates were dissolved in a 1:4 ratio of acetonitrile to DMSO such that the final assay buffer consisted of $1 \%(\mathrm{v} / \mathrm{v})$ acetonitrile and $4 \%(\mathrm{v} / \mathrm{v})$ DMSO in addition to $0.2 \%(\mathrm{v} / \mathrm{v})$ TritonX-100, $20 \mathrm{mM}$ HEPES, and $1 \mathrm{mM}$ EDTA at $\mathrm{pH}$ 7.5. For consistency, kinetics for the $\mathrm{C} 2$ and $\mathrm{C} 4$ substrates were also measured in this manner. Extinction coefficients used were 12790 and $11869 \mathrm{M}^{-1} \mathrm{~cm}^{-1}$, respectively with a path length of $0.55 \mathrm{~cm}$ when the reactions were done in 96-well plates. Kinetic constants $V_{\max }$ and $K_{\mathrm{m}}$ were obtained by fitting the experimental data to the Michaelis-Menten equation. ${ }^{31}$ All kinetic data was obtained in triplicate; the errors are the standard deviation of the data sets but could be underestimated since the data was from the same sample preparation.

Crystallization. Crystallization of native DLH was done using conditions already reported. ${ }^{24}$ The variants crystallized under similar conditions, although some required microseeding or slightly altered precipitant concentration (0.8-1.2 $\mathrm{M}$ ammonium sulfate).

Data Collection and Processing. Diffraction data was collected using $\mathrm{Cu} \mathrm{K} \alpha$ radiation at a temperature of $100 \mathrm{~K}$. Data were recorded as a contiguous sequence of half-degree rotations using a MAR345 image plate detector mounted on a rotating anode Rigaku RA Micro 7 HFM X-ray generator with cooling supplied by an Oxford Cryostream 700 series. Between 225 and 480 frames were collected for each crystal with $180 \mathrm{~s}$ exposure time per frame for all except the S-3 and B-4 variant crystals, which required $300 \mathrm{~s}$ exposure time per frame. Data collection statistics are listed in Table S6, Supporting Information.

Structure Determination and Refinement. The native structure was determined by refining it against a known DLH structure (PDB entry 1ZI6). ${ }^{23}$ All variant structures were first subjected to a rigid body cycle, followed by a cyclical manual inspection of electron density maps using COOT, ${ }^{32}$ and then further refined using REFMAC5 $5^{33}$ in the CCP4 suite of programs. ${ }^{34}$ The average error in the native coordinates was found to be $0.09 \AA$ as determined by the diffraction-component precision index (DPI) value for the model, while the corresponding errors for the A-6, S-2, S-3, B-1, and B-4 variant structures are 0.13, 0.08, $0.09,0.10$, and $0.12 \AA$ respectively. ${ }^{35}$ Structures were superimposed using SUPERPOSE. ${ }^{36}$ 
Substrate Docking. The crystal structures of the native and variant enzymes were first relaxed using the ROSETTA 3.5 relax application to minimize internal clashes and further refine the structure. ${ }^{37}$ Twenty structures were generated for each enzyme, and the lowest total scoring structure was used as input into the docking protocol. Rotamers were generated for $p$-nitrophenyl acetate and $p$-nitrophenyl butyrate using balloon version 1.4.1.1068. ${ }^{38}$ The ligands were constrained in the active site with distance and angle restraints. Substrate docking was done using Rosettascripts 3.5 and ligand dock.xml file with commands -no_optH, -ex1, -exlaro, and -ex2. ${ }^{39}$ Five hundred structures were generated from the docking protocol, and the structures were filtered by ligand RMSD and interface energy. The top $10 \%$ of lowest scoring structures were analyzed visually using PyMol. ${ }^{40}$

\section{ASSOCIATED CONTENT}

\section{S Supporting Information}

Additional figures, evolution tables, and detailed methods. This material is available free of charge via the Internet at http://pubs.acs.org.

\section{Accession Codes}

Coordinates for the native and variant structures have been deposited into the Protein Data Bank. Their PDB codes are 4U2B (native), 4U2C (A-6), 4U2D (S-2), 4U2E (S-3), 4U2F (B-1), and $4 \mathrm{U} 2 \mathrm{G}$ (B-4).

\section{AUTHOR INFORMATION}

\section{Corresponding Authors}

*E-mail: jo.porter@anu.edu.au.

*E-mail: david.ollis@anu.edu.au.

\section{Notes}

The authors declare no competing financial interest.

\section{ACKNOWLEDGMENTS}

We acknowledge the Australian Research Council for funding and the staff at the BRF for performing the DNA sequencing. J.P. is supported by an Australian Postgraduate Award from the Australian government.

\section{REFERENCES}

(1) Turner, N. J. (2009) Directed evolution drives the next generation of biocatalysts. Nat. Chem. Biol. 5, 567-573.

(2) Jackson, C. J., Gillam, E. M. J., and Ollis, D. L. (2010) Directed Evolution of Enzymes, in Comprehensive Natural Products II (Liu, H.W., and Mander, L., Eds.), pp 723-749, Elsevier, Oxford.

(3) Woodyer, R., Chen, W., and Zhao, H. (2004) Outrunning nature: Directed evolution of superior biocatalysts. J. Chem. Educ. 81, 126133.

(4) Dalby, P. A. (2011) Strategy and success for the directed evolution of enzymes. Curr. Opin. Struct. Biol. 21, 473-480.

(5) Ollis, D. L., Cheah, E., Cygler, M., Dijkstra, B., Frolow, F., Franken, S. M., Harel, M., Remington, S. J., Silman, I., Schrag, J., Sussman, J. L., Verschueren, K. H. G., and Goldman, A. (1992) The alpha /beta hydrolase fold. Protein Eng. 5, 197-211.

(6) Carr, P. D., and Ollis, D. L. (2009) $\alpha / \beta$ hydrolase fold: An update. Protein Pept. Lett. 16, 1137-1148.

(7) Heikinheimo, P., Goldman, A., Jeffries, C., and Ollis, D. L. (1999) Of barn owls and bankers: A lush variety of $\alpha / \beta$ hydrolases. Structure 7, R141-R146.

(8) Pathak, D., Ngai, K. L., and Ollis, D. (1988) X-ray crystallographic structure of dienelactone hydrolase at 2.8 Å. J. Mol. Biol. 204, 435-445.

(9) Pathak, D., and Ollis, D. (1990) Refined structure of dienelactone hydrolase at 1.8 Å. J. Mol. Biol. 214, 497-525.
(10) Ollis, D. L., and Ngai, K. L. (1985) Crystallization and preliminary X-ray crystallographic data of dienelactone hydrolase from Pseudomonas sp. B13. J. Biol. Chem. 260, 9818-9819.

(11) Schmidt, E., and Knackmuss, H. J. (1980) Chemical structure and biodegradability of halogenated aromatic compounds. Conversion of chlorinated muconic acids into maleoylacetic acid. Biochem. J. 192, 339-330.

(12) Ngai, K. L., Schloemann, M., Knackmuss, J., and Ornston, L. N. (1987) Dienelactone hydrolase from Pseudomonas sp. strain B13. J. Bacteriol. 169, 699-703.

(13) Harwood, C. S., and Parales, R. E. (1996) The $\beta$-ketoadipate pathway and the biology of self-identity. Annu. Rev. Microbiol. 50, 553590.

(14) Stanier, R. Y., and Ornston, L. N. (1973) The $\beta$-ketoadipate pathway. Adv. Microb. Physiol. 9, 89-151.

(15) Pathak, D., Ashley, G., and Ollis, D. (1991) Thiol protease-like active site found in the enzyme dienelactone hydrolase: Localization using biochemical, genetic, and structural tools. Proteins Struct., Funct., Genet. 9, 267-279.

(16) Walker, I., Easton, C. J., and Ollis, D. L. (2000) Site-directed mutagenesis of dienelactone hydrolase produces dienelactone isomerase. Chem. Commun. (Cambridge, U. K.), 671-672.

(17) Walker, I., Hennessy, J. E., Ollis, D. L., and Easton, C. J. (2012) Substrate-Induced Conformational Change and Isomerase Activity of Dienelactone Hydrolase and its Site-Specific Mutants. ChemBioChem 13, 1645-1651.

(18) Strohmeier, G. A., Pichler, H., May, O., and Gruber-Khadjawi, M. (2011) Application of Designed Enzymes in Organic Synthesis. Chem. Rev. 111, 4141-4164.

(19) Bornscheuer, U. T., Huisman, G. W., Kazlauskas, R. J., Lutz, S., Moore, J. C., and Robins, K. (2012) Engineering the third wave of biocatalysis. Nature 485, 185-194.

(20) Bloom, J. D., Silberg, J. J., Wilke, C. O., Drummond, D. A., Adami, C., and Arnold, F. H. (2005) Thermodynamic prediction of protein neutrality. Proc. Natl. Acad. Sci. U. S. A. 102, 606-611.

(21) Bloom, J. D., Labthavikul, S. T., Otey, C. R., and Arnold, F. H. (2006) Protein stability promotes evolvability. Proc. Natl. Acad. Sci. U. S. A. $103,5869-5874$.

(22) Tokuriki, N., and Tawfik, D. S. (2009) Stability effects of mutations and protein evolvability. Curr. Opin. Struct. Biol. 19, 596604.

(23) Kim, H. K., Liu, J. W., Carr, P. D., and Ollis, D. L. (2005) Following directed evolution with crystallography: Structural changes observed in changing the substrate specificity of dienelactone hydrolase. Acta Crystallogr., Sect. D: Biol. Crystallogr. D61, 920-931.

(24) Porter, J. L., Carr, P. D., Collyer, C. A., and Ollis, D. L. (2014) Crystallization of dienelactone hydrolase in two space groups: structural changes caused by crystal packing. Acta Crystallogr., Sect. F: Struct. Biol. Cryst. Commun. 70, 884-889.

(25) Kasberg, T., Seibert, V., Schloemann, M., and Reineke, W. (1997) Cloning, characterization, and sequence analysis of the clcE gene encoding the maleylacetate reductase of Pseudomonas sp. strain B13. J. Bacteriol. 179, 3801-3803.

(26) Cheah, E., Ashley, G. W., Gary, J., and Ollis, D. (1993) Catalysis by dienelactone hydrolase: A variation on the protease mechanism. Proteins Struct., Funct., Genet. 16, 64-78.

(27) Cheah, E., Austin, C., Ashley, G. W., and Ollis, D. (1993) Substrate-induced activation of dienelactone hydrolase: An enzyme with a naturally occurring Cys-His-Asp triad. Protein Eng. 6, 575-583.

(28) Sussman, J. L., Harel, M., Frolow, F., Oefner, C., Goldman, A., Toker, L., and Silman, I. (1991) Atomic structure of acetylcholinesterase from Torpedo californica: A prototypic acetylcholine-binding protein. Science 253, 872-879.

(29) Yang, H., Carr, P., McLoughlin, S. Y., Liu, J., Horne, I., Qiu, X., Jeffries, C. M. J., Russell, R., Oakeshott, J., and Ollis, D. (2003) Evolution of an organophosphate-degrading enzyme: a comparison of natural and directed evolution. Protein Eng., Des. Sel. 16, 135-135. 
(30) Stevenson, B. J., Liu, J.-W., and Ollis, D. L. (2008) Directed Evolution of Yeast Pyruvate Decarboxylase 1 for Attenuated Regulation and Increased Stability. Biochemistry 47, 3013-3025.

(31) Johnson, K. A., and Goody, R. S. (2011) The Original Michaelis Constant: Translation of the 1913 Michaelis-Menten Paper. Biochemistry 50, 8264-8269.

(32) Emsley, P., and Cowtan, K. (2004) Coot: Model-building tools for molecular graphics. Acta Crystallogr., Sect. D: Biol. Crystallogr. 60, $2126-2132$.

(33) Murshudov, G. N., Vagin, A. A., and Dodson, E. J. (1997) Refinement of Macromolecular Structures by the Maximum-Likelihood Method. Acta Crystallogr., Sect. D: Biol. Crystallogr. 53, 240255.

(34) Winn, M. D., Ballard, C. C., Cowtan, K. D., Dodson, E. J., Emsley, P., Evans, P. R., Keegan, R. M., Krissinel, E. B., Leslie, A. G. W., McCoy, A., McNicholas, S. J., Murshudov, G. N., Pannu, N. S., Potterton, E. A., Powell, H. R., Read, R. J., Vagin, A., and Wilson, K. S. (2011) Overview of the CCP4 suite and current developments. Acta Crystallogr., Sect. D: Biol. Crystallogr. 67, 235-242.

(35) Cruickshank, D. (1999) Remarks about protein structure precision. Acta Crystallogr., Sect. D: Biol. Crystallogr. 55, 583-601.

(36) Krissinel, E., and Henrick, K. (2004) Secondary-structure matching (SSM), a new tool for fast protein structure alignment in three dimensions. Acta Crystallogr., Sect. D: Biol. Crystallogr. 60, 22562268.

(37) Raman, S., Vernon, R., Thompson, J., Tyka, M., Sadreyev, R., Pei, J., Kim, D., Kellogg, E., DiMaio, F., Lange, O., Kinch, L., Sheffler, W., Kim, B.-H., Das, R., Grishin, N. V., and Baker, D. (2009) Structure prediction for CASP8 with all-atom refinement using Rosetta. Proteins: Struct., Funct., Bioinf. 77, 89-99.

(38) Vainio, M. J., and Johnson, M. S. (2007) Generating Conformer Ensembles Using a Multiobjective Genetic Algorithm. J. Chem. Inf. Model. 47, 2462-2474.

(39) Lemmon, G., and Meiler, J. (2012) Rosetta Ligand Docking with Flexible XML Protocols, in Computational Drug Discovery and Design (Baron, R., Ed.), pp 143-155, Springer, New York.

(40) (2010) The PyMOL Molecular Graphics System, version 1.4.1, Schrodinger, LLC. 